\title{
RINOPLASTÍA DEFINITIVA EN DEFORMIDADES NASOLABIALES DE PACIENTES FISURADOS: VALORACIÓN ESTÉTICA DE LA EXPERIENCIA QUIRÚRGICA*
}

\author{
Drs. Carlos Giugliano V., ${ }^{1,3}$, Andrea Hasbún N. ${ }^{2,3}$, \\ José Tomás Gantz V. ${ }^{4}$, Enrique Hanuch V. ${ }^{5}$ \\ ${ }^{1}$ Fundación Alfredo Gantz Mann. Hospital del Niño con Fisura. Universidad de Chile. \\ 2 Hospital Dr. Roberto del Río, Universidad de Chile. \\ 3 Cirugía Plástica, Clínica Alemana. \\ 4 Servicio de Cirugía, Hospital Padre Hurtado. \\ 5 Otorrinolaringología, Clínica Alemana. \\ Santiago, Chile.
}

\begin{abstract}
\section{Definitive rhinoplasty in secondary deformities of the cleft patients: esthetical evaluation of the surgical experience}

Introduction: The three dimensional structure of the nose, heterogenia composition of tissues (bone, cartilage and soft tissue) and the presence of previous surgery in the nasolabial territory makes definitive rhinoplasty very difficult in cleft patients. It is therefore essential to analyze the results obtained after tertiary or definitive rhinoplasty in these patients. Objective: To present the aesthetic results obtained through an objective method and supported in the literature; cleft patients undergoing definitive rhinoseptoplasty. Methods: Postoperative photographic analysis of all cleft patients undergoing definitive rhinoplasty between January 1, 2010 and July 31, 2014 was performed by 4 reviewers blinded to study. Results: Tertiary rhinoseptoplasty was conducted in 54 patients with a mean age of 21 years (range 14 to 51 years), of whom 24 $(48.7 \%)$ were female and $30(51.2 \%)$ male. $36(66 \%)$ patients had unilateral cleft and $18(33 \%)$ were patients with bilateral cleft. The average assessments using the Score Strasser believes that $29.6 \%$ (16) cases showed excellent results, $57.4 \%$ (31) cases in the good score and $12.9 \%$ (7) classified in mediocre settings. There were no cases with bad score. Conclusion: The results of this study allow us to propose a learning curve enough to obtain comparable or superior to those presented in this casuistry safe, functional and aesthetic result.

Key words: Rhinoplasty, cleft, Evaluation.

\footnotetext{
*Recibido el 17 de junio de 2015 y aceptado para publicación el 19 de agosto de 2015.

*Los autores del presente trabajo declaran no tener ningún tipo de conflicto de interés, así como tampoco subvención para la realización del mismo.
}

Correspondencia: Dr. Carlos Giugliano V. cgiugliano@alemana.cl 


\section{Resumen}

Introducción: La estructura tridimensional de la nariz, la composición heterogénea de sus tejidos (hueso, cartílago y partes blandas) y la presencia muchas veces de cirugías previas en el territorio naso labial hace que la reparación definitiva de ésta sea de gran dificultad en el paciente fisurado. Resulta entonces fundamental analizar los resultados que se obtienen tras una rinoplastía definitiva en estos pacientes. Objetivo: Presentar los resultados estéticos, obtenidos a través de un método objetivo y avalado en la literatura; de pacientes fisurados sometidos a una rinoseptoplastía definitiva. Material y Método: Se realizó análisis fotográfico postoperatorio de la totalidad de pacientes fisurados sometidos a rinoplastía definitiva entre el 1 de enero de 2010 y 31 de julio de 2014, por 4 evaluadores ciegos al estudio. Resultados: Se realizó rinoseptoplastía definitiva en 54 pacientes, con edad promedio de 21 años (rango entre 14 y 51 años), de los cuales 24 (48,7\%) correspondían a sexo femenino y $30(51,2 \%)$ a sexo masculino. Treinta y seis $(66 \%)$ pacientes presentaban fisura unilateral (LLU) y 18 (33\%) correspondían a pacientes con fisura bilateral (LLB). El promedio de evaluaciones, utilizando el Score de Strasser, considera que el 29,6\% (16) de los casos presentaba resultados excelentes, $57,4 \%$ (31) de los casos dentro del score bueno y $12,9 \%$ (7) clasificaba dentro de los casos mediocres. No hubo casos con puntaje malo. Conclusión: Los resultados del presente trabajo, nos permite proponer una curva de aprendizaje suficiente que permita obtener resultados seguros, funcionales y estéticos comparables o superiores a los presentados en esta casuística.

Palabras clave: Rinoplastía, fisurados, evaluación.

\section{Introducción}

La reparación de la deformidad nasal resulta un gran desafío en pacientes fisurados. La estructura tridimensional de la nariz, la composición heterogénea de sus tejidos (hueso, cartílago y partes blandas) hace que la reparación definitiva de ésta sea de gran dificultad para el cirujano. La correcta reparación nasal a través de una metodología sistematizada en su manejo quirúrgico, requiere de la evaluación previa de las diferentes anomalías asociadas, así como sus efectos en la fisiología de la zona facial comprometida.

La deformidad nasal secundaria, está influenciada por las características de la lesión primaria (fisura uni o bilateral), el crecimiento facial y las intervenciones anteriores en la zona nasolabial ${ }^{1-3}$. La nariz es una unidad estético funcional. Deformidades secundarias en pacientes fisurados que han completado su crecimiento, afectan gravemente sus relaciones, su desarrollo social y laboral. Esta es la razón por la cual la rinoplastía secundaria o definitiva implica la corrección, durante la edad adulta, de las rinodeformidades residuales de los diferentes procedimientos que se realizan en el contexto del tratamiento integral del paciente con fisura. Esta cirugía contempla el manejo definitivo de la pirámide nasal en su estructura osteo-cartilaginosa, con criterios funcionales y estéticos ${ }^{4}$.

El propósito del presente estudio es analizar los resultados estéticos, a través de un método objetivo y avalado en la literatura, de pacientes fisurados sometidos a una rinoseptoplastía definitiva. Se presenta además el protocolo de evaluación preoperato- rio, técnicas quirúrgicas y seguimiento de pacientes fisurados sometidos a rinoplastía definitiva.

\section{Material y Métodos}

Se realizó el análisis fotográfico postoperatorio de la totalidad de pacientes fisurados sometidos a rinoplastía definitiva entre el 1 de enero de 2010 y 31 de julio de 2014. En total, 54 pacientes fisurados fueron sometidos a una rinoplastía secundaria por el mismo cirujano (C.G.V). El seguimiento de los pacientes incluye registro fotográfico postoperatorio inmediato, a los 7 días posterior al procedimiento y alejado (6 meses). Para llevar a cabo el análisis de los resultados estéticos, se solicitó la participación de cuatro cirujanos plásticos (ciegos al estudio) quienes evaluaron las fotografías de todos los pacientes de la serie. Se usaron proyecciones fotográficas del control alejado, en antero-posterior, lateral, tres cuartos e inferiores. Se utilizó la escala de Stras$\operatorname{ser}^{5,6}$ para la evaluación de las mismas, que clasifica los resultados como excelente (puntuación 0), buena (puntuación 1-4), mediocre (puntuación 5-14) y malo (puntuación $>15$ ). Todas las cirugías fueron realizadas bajo anestesia general. La técnica utilizada fue la rinoplastía abierta, previa infiltración de solución de epinefrina 1:50.000. La cirugía comienza con la etapa funcional (tabique y cornetes). Para el manejo de la subunidad punta nasal, se realiza mediante resección cefálica, la simetrización de los cartílagos alares. La reconstrucción del neocomplejo alar se lleva a cabo con material monofilamento irreabsorbible 5/0. El siguiente paso es la plastía de 
la base de los alares y la regularización del tamaño de la(s) fosa(s) nasal(es).

La etapa estética comienza con la resección de la giba osteo cartilaginosa, osteotomía lateral externa y según necesidad uso de injertos de cartílago. No se usa ningún injerto óseo o material aloplástico. El abordaje se cierra usando un plano transcolumelar con material monofilamento irreabsorbible $6 / 0$.

En el postoperatorio inmediato se utiliza férula en el dorso nasal (moldaje térmico) durante siete días y una férula septal (de fluoroplástico) durante 15 días. Tampones endonasales entre 2 y 4 días. Para manejo de las narinas se utiliza conformador nasal durante 6 meses (Silimed ${ }^{\mathrm{MR}}$ ).

Ante la necesidad de retoque labial, la cirugía se define de acuerdo con el o los defectos específico(s) encontrado(s). Para el manejo del labio corto, se realiza la reoperación completa o plastía simple para elongación del mismo, por lo general con colgajos triangulares. En los casos de labios largos o las áreas con exceso de volumen, se realizan técnicas reductivas. Cuando el labio es adecuado del punto de vista estético y funcional la revisión de cicatriz fue el único procedimiento realizado.

\section{Resultados}

Se realizó rinoseptoplastía definitiva en 54 pacientes, con edad promedio de 21 años (rango entre 14 y 51 años), de los cuales $24(48,7 \%)$ correspondían a sexo femenino y $30(51,2 \%)$ a sexo masculino. Treinta y seis $(66 \%)$ pacientes presentaban fisura unilateral (LLU) y 18 (33\%) correspondían a pacientes con fisura bilateral (LLB).

Todos los pacientes de la serie fueron sometidos a rinoplastía definitiva, de los cuales 17 casos $(31,4 \%)$ tenían el antecedente de rinoplastía previa. El 77,7\% del total de pacientes (42), requirió una cirugía asociada a la rinoplastía. El desglose de los diferentes procedimientos utilizados en la rinoplastía definitiva corresponde a osteotomía en 46 casos $(85 \%)$, turbinectomía en 30 casos $(55,5 \%)$, septoplastía 39 pacientes $(72,2 \%)$, injerto de cartílago 52 casos $(96,2 \%)$ y plastía de la base de los alares en 44 pacientes $(81,4 \%)$. Los mismos procedimientos, según tipo de fisura se presentan en la Tabla 1.

Cincuenta y dos pacientes de la serie requirieron injerto de cartílago. De ellos, la zona dadora correspondió en el 90,7\% (49) de los casos al tabique nasal, el dorso cartilaginoso en 3 casos $(5,5 \%)$ y cartílago alar contralateral al lado de la fisura en 1 caso $(1,8 \%)$. Respecto a la utilización de dichos injertos, el 92,5\% (51) de los casos se utilizó como vástago columelar, en el 46,2\% (25) para proyección de la punta nasal y en el 9,2\% (5) se utilizó como relleno en la base alar y el piso nasal. Por último sólo en 1 paciente se utilizó dicho cartílago en injertos tipo Spreader $(1,8 \%)$ para camuflar una laterorrinia y no con fines funcionales.

En 44 pacientes $(81,5 \%)$ se realizó plastía de la base del o los cartílagos alares. La técnica utilizada en dichos casos, correspondió en 21 pacientes $(58,8 \%)$ a colgajo de avance tipo V-Y, colgajo tipo semilunar en 22 casos $(40,7 \%)$ y adelgazamiento de la base alar en 1 caso $(1,8 \%)$.

Las cirugías asociadas fueron la revisión de labios en 39 casos $(92,8 \%)$, cierre de la hendidura palatina en 2 casos y una plastía vestibular.

Tres pacientes $(5,5 \%)$ presentaron algún tipo de complicación. Estas correspondieron a una perforación septal y 2 dehiscencias columelares. No hubo complicaciones en cirugías asociadas.

El promedio de las observaciones realizadas por los 4 evaluadores consideran que el 29,6\% (16) de los casos presentaba resultados excelentes, $57,4 \%$ (31) de los casos dentro del score bueno y $12,9 \%$ (7) clasificaba dentro de los casos mediocres. No hubo casos con puntaje malo. En las Figuras 1 y 2 se presentan los resultados pre y postoperatorios alejados, de la misma forma en que fueron presentados a los evaluadores del estudio. En la Tabla 2, Figuras 1 y 2 , se presentan los resultados del análisis estético general y según el tipo de fisura y presencia de rinoplastía previa respectivamente.

Tabla 1. Técnica quirúrgica según tipo de Fisura

\begin{tabular}{|lcr|}
\hline Técnica quirúrgica & $\begin{array}{c}\text { LLU } \\
(\mathbf{n}=\mathbf{3 6})\end{array}$ & $\begin{array}{c}\text { LLB } \\
(\mathbf{n}=\mathbf{1 8})\end{array}$ \\
\hline Turbinectomía & $23(63,8 \%)$ & $7(38,8 \%)$ \\
Septoplastía & $28(77,7 \%)$ & $11(61,1 \%)$ \\
Queiloplastía & $25(69,4 \%)$ & $14(77,7 \%)$ \\
Plastía alar & $29(80,5 \%)$ & $15(83,3 \%)$ \\
Injerto cartílago & $35(97,2 \%)$ & $17(94,4 \%)$ \\
Rinoplastía definitiva previa & $9(25 \%)$ & $8(44,4 \%)$ \\
\hline
\end{tabular}

Tabla 2. Resultado Score Strasser $(n=54)$

\begin{tabular}{|lr|}
\hline Score Strasser & \\
\hline 0 (Excelente) & $16(29,6 \%)$ \\
1 a 4 (Bueno) & $31(57,4 \%)$ \\
5 a 14 (Mediocre) & $7(12,9 \%)$ \\
$>$ a 15 (Malo) & $0(0,0 \%)$ \\
\hline
\end{tabular}



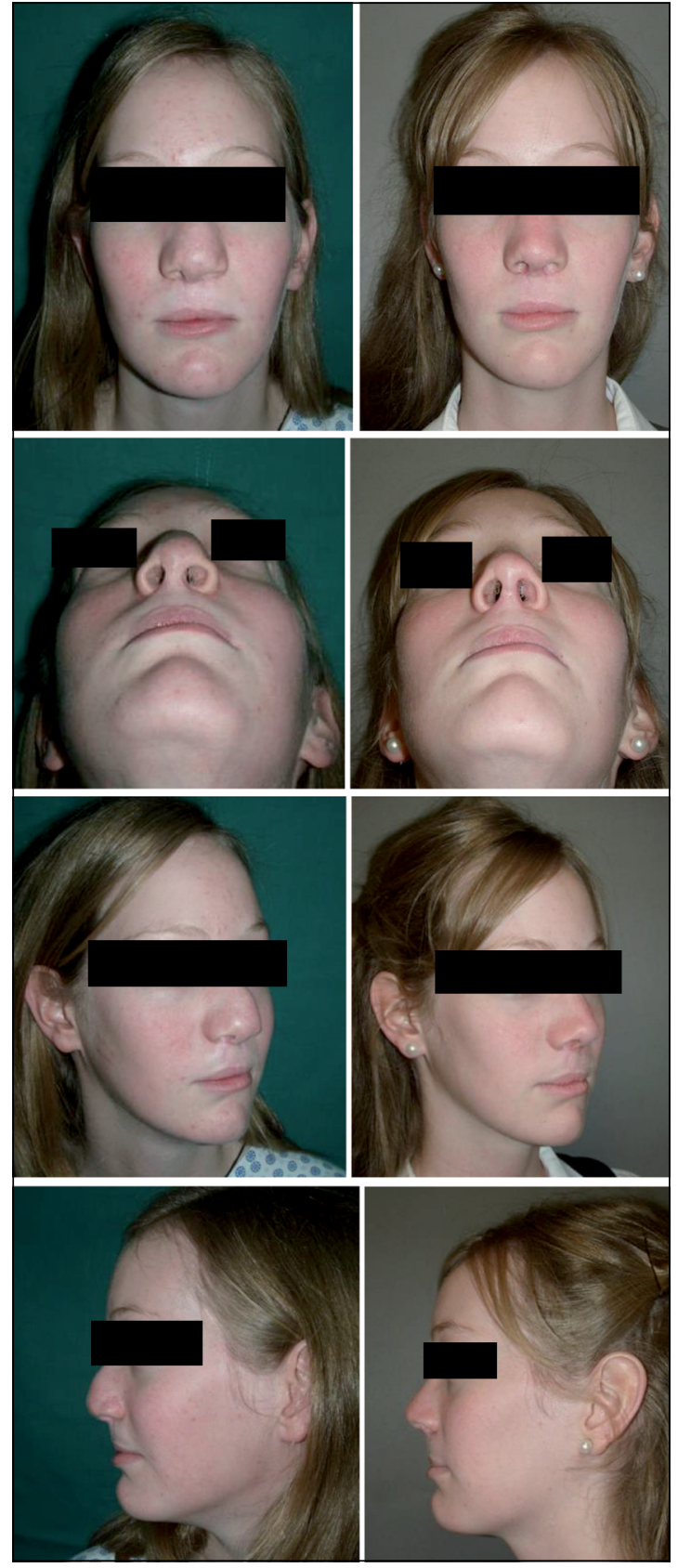

Figura 1. Score de Strasser según tipo de fisura.

\section{Discusión}

Nuestro protocolo para el manejo quirúrgico del fisurado, contempla entre los tres y seis meses la queiloplastía con rinoplastía primaria en el mismo tiempo operatorio. A los 5 años, previo a la edad de inicio escolar, se realiza retoque naso labial (rino queiloplastía intermedia) en aquellos pacientes que
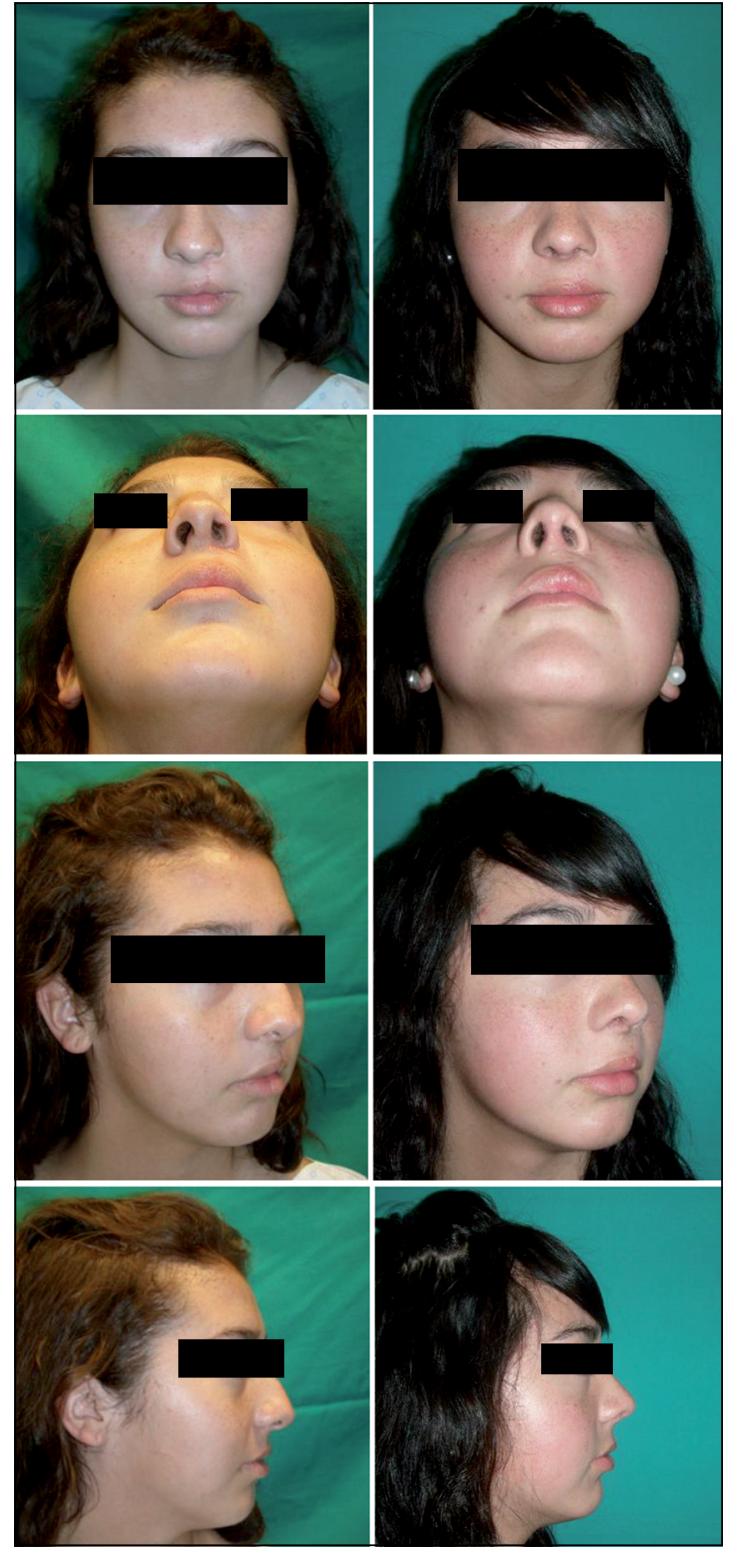

Figura 2. Score de Strasser según presencia o ausencia de rinoplastía previa.

presenten obstrucción nasal severa determinada por desviación septal, rinodeformidad no resulta con el procedimiento primario o por necesidad psicológica del niño. Estas indicaciones han sido ampliamente discutidas en la literatura ${ }^{7,8}$ y se encuentran en concordancia con el protocolo anteriormente descrito. La rinoplastía definitiva la realizamos entre los 14 y 18 años una vez que el período del crecimiento facial ya se ha completado. Esta cirugía constituye el último evento quirúrgico, por parte del cirujano 
plástico en el manejo del paciente fisurado. Generalmente esta cirugía se asocia a la última revisión del labio según necesidad como lo hemos señalado en el presente trabajo.

La literatura es clara en mencionar que la Rinoplastía definitiva debe ser realizada una vez se halla completado el proceso de crecimiento maxilar y nasal. El manejo quirúrgico del maxilar mediante una cirugía ortognática, según nuestro protocolo es requisito previo a la rinoplastía definitiva. En esta etapa del desarrollo y de la evolución clínica de este tipo de pacientes, presentan un alto nivel de expectativas toda vez que la mayoría de ellos son adolescentes o jóvenes, disconformes con el estigma de la nariz fisurada. La dispersión etaria encontrada en nuestra casuística, obedece entre otras cosas, a la necesidad de efectuar la rinoplastía definitiva en pacientes que han sido derivados al cirujano, fuera de los plazos establecidos en el protocolo descrito anteriormente. Este hecho no los privó de la rinoplastía definitiva, así como tampoco estos pacientes fueron excluidos en el análisis objetivo de resultados estéticos aquí presentados.

Resultados estéticos post rinoplastía definitiva se han estudiado a través de la evaluación subjetiva de pacientes y sus cirujanos. Algunos estudios han analizado los resultados estéticos utilizando fotografías $^{9-11}$ preoperatoria y postoperatoria, así como el uso de nuevas tecnologías como la exploración en 3 dimensiones a través del Scanner superficial con láser para evaluar los cambios del contorno nasal post quirúrgico ${ }^{12,13}$. La utilidad de la objetivación de los resultados estéticos postoperatorios, tendría gran utilidad a la hora de entender los efectos y sus resultados en determinadas técnicas quirúrgicas. Por ejemplo, Pitak-Arnnop y cols. ${ }^{9}$, compararon las percepciones de los pacientes que fueron sometidos a una rinoplastía secundaria con o sin injerto columelar. En dicha experiencia se encontró que el injerto columelar se asocia a un mejor resultado estético en comparación a aquellos que no se usó dicho injerto, situación que no pudo ser comprobada en nuestra experiencia. Nuestra serie, a través del Score de Strasser, avalado en la literatura para objetivar resultados estéticos en cirugía plástica, nos parece útil para el análisis de los resultados cosméticos post rinoplastía secundaria en pacientes fisurados. Los resultados del presente estudio no pueden ser comparados con los escasos trabajos existentes en la literatura, puesto que la metodología de los mismos no es comparable. Nuestra serie, logra demostrar que el $87 \%$ de los pacientes tuvo resultados igual o mejor que buenos. Sólo el 12,9\% de los casos, fue clasificado como mediocre. Cuando se realiza el desglose de los resultados según la presencia de rinoplastía previa, los resultados se mantienen en la misma tendencia. Los pacientes con fisura bilateral de labio se asocian a peores resultados estéticos que aquellos que presentaban fisura unilateral.

Si bien el presente reporte no evalúa los resultados funcionales, nuestro protocolo incluye siempre, los aspectos funcionales de esta cirugía (tabique y turbinectomía), mejorando paralelamente de esta forma el aspecto estético comprometido por la desviación septal cuando está presente, la asimetría de las narinas y de las bases alares. De esta forma, en nuestra casuística, se realizó turbinectomía en el $55,5 \%$ y septoplastía en el $72,2 \%$.

En nuestra serie en el 96,2\% de los casos, al igual que lo reportado en la literatura ${ }^{14,15,17}$, utilizamos injerto de cartílago. Guyuron señala que dependiendo del grado de hipoplasia de los tejidos, se puede utilizar injertos de cartílago o compuestos, especialmente en el soporte columelar para la proyección de la punta nasal y la construcción de un marco nasal que imite la apariencia de un cartílago lateral normal ${ }^{18}$. El componente de aumento nasal habitualmente se refiere a mejorar la proyección de la punta nasal y el complejo alar. En nuestra serie se destaca el vástago columelar en la mayoría de los casos $(92,5 \%)$ y en casi la mitad de ellos el uso de injerto de la punta nasal $(46,2 \%)$. El septo nasal, al igual que lo reportado por Bashir M. y cols., es el principal sitio donante de cartílago.

Flores y col., en su experiencia de 10 años de seguimiento, definen el abordaje con rinoplastía abierta como el método de elección en estos casos, en concordancia con la mayoría de los reportes de la literatura. Este abordaje permite un manejo adecuado y bajo visión directa de la punta nasal, la que por lo general presenta gran distorsión de la anatomía y cicatrices previas. El protocolo presentado en el presente trabajo contempla el abordaje abierto en la totalidad de los casos.

El aspecto reductivo es muy frecuente en nuestra casuística, requiriendo en un $85 \%$ de los casos manejo de pirámide ósea y dorso nasal. A nivel del complejo alar a veces es necesario reducir tamaño de los cartílagos en busca de la simetría especialmente en los fisurados unilaterales, lo que se encuentra en concordancia con los diversos reportes de la literatura ${ }^{19-21}$.

La planificación quirúrgica debe ser individual, no existiendo una técnica estándar. La deformidad nasal del paciente con fisura es técnicamente compleja, la evaluación debe considerar aspectos anatómicos, funcionales y cicatriciales por cirugías previas.

La rinoplastía definitiva en el paciente fisurado es una cirugía más prolongada que la rinoplastía estética clásica por su complejidad y porque frecuentemente requiere de otros procedimientos 
complementarios, en nuestra serie el $92,8 \%$ de los pacientes. Lo anteriormente señalado nos permite plantear que el cirujano debe realizar una curva de aprendizaje suficiente que le permita obtener resultados seguros, funcionales y estéticos comparables o superiores a los presentados en nuestra casuística.

\section{Referencias}

1. Sykes JM, Jang YJ. Cleft lip rhinoplasty. Facial Plast Surg Clin North Am. 2009;17:133-44, vii.

2. Shih CW, Sykes JM. Correction of the cleft-lip nasal deformity. Facial Plast Surg. 2002;18:253-62.

3. Lee TS, Schwartz GM, Tatum SA. Rhinoplasty for cleft and hemangioma-related nasal deformities. Curr Opin Otolaryngol Head Neck Surg. 2010;18:526-35.

4. Cutting CB. Secondary cleft lip nasal reconstruction: state of the art. Cleft Palate Craniofac J. 2000;37:53841.

5. Strasser EJ. Application of an Objective Grading System for the Evaluation of Cosmetic Surgical Results. Plast Reconstr Surgery 1999;104:2282-5.

6. Strasser EJ. Application o fan objective Trading system for evaluation of cosmetic surgical results. Plast Reconstr Surg. 2002;109:1733-40.

7. Giugliano C. Manejo quirúrgico de la fisura labiopalatina. En: Rostion CG. Cirugía Pediátrica 2a Edición. Santiago: Publicaciones técnicas Mediterráneo, 2014;394411.

8. Shih CW, Sykes JM. Correction of the cleft-lip nasal deformity. Facial Plast Surg. 2002;18:253-62.

9. Pitak-Arnnop P, Hemprich A, Dhanuthai K, Yildirim V, Pausch NC. Panel and patient perceptions of nasal aesthetics after secondary cleft rhinoplasty with versus without columellar grafting. J Craniomaxillofac Surg. 2011;39:319-25.

10. Chaithanyaa N, Rai KK, Shivakumar HR, Upasi A. Evaluation of the outcome of secondary rhinoplasty in cleft lip and palate patients. J Plast Reconstr Aesthet Surg. 2011;64:27-33.
11. Huempfner-Hierl H, Hemprich A, Hierl T. Results of a prospective anthropometric and functional study about aesthetics and nasal respiration after secondary rhinoplasty in cleft lip and palate patients. J Craniofac Surg. 2009;20(suppl 2):1863-75.

12. Nakamura N, Sasaguri M, Okawachi T, Nishihara K, Nozoe E. Secondary correction of bilateral cleft lip nose deformity: clinical and three-dimensional observations on pre-and postoperative outcome. J Craniomaxillofac Surg. 2011;39:305-12.

13. Okawachi T, Nozoe E, Nishihara K, Nakamura N. 3-dimensional analyses of outcomes following secondary treatment of unilateral cleft lip nose deformity. J Oral Maxillofac Surg. 2011;69:322-32.

14. Bashir M, Malik A, Khan FA. Comparison of suture and graft techniques in secondary unilateral cleft rhinoplasty. J Craniofac Surg. 2011;22:2172-5.

15. Hwang K, Kim HJ, Paik MH. Unilateral cleft nasal deformity correction using conchal cartilage lily flower graft. J Craniofac Surg. 2012;23:1770-2.

16. Masuoka H, Kawai K, Morimoto N, Yamawaki S, Suzuki S. Open rhinoplasty using conchal cartilage during childhood to correct unilateral cleft-lip nasal deformities. J Plast Reconstr Aesthet Surg. 2012;65:857-63.

17. Turkaslan T, Turan A, Yogun N, Ozsoy Z. A novel approach to cleft lip nose deformity: posterior dome graft technique. J Craniofac Surg. 2008;19:1359-63.

18. Guyuron B. MOC-PS(SM) CME article: late cleft lip nasal deformity. Plast Reconstr Surg. 2008;121(suppl):1-11.

19. Flores RL, Sailon AM, Cutting CB. A novel cleft rhinoplasty procedure combining an open rhinoplasty with the Dibbell and Tajima techniques: a 10-year review. Plast Reconstr Surg. 2009;124:2041-7.

20. Bashir M, Malik A, Khan FA. Comparison of suture and graft techniques in secondary unilateral cleft rhinoplasty. J Craniofac Surg. 2011;22:2172-5.

21. Stal S, Hollier L. Correction of secondary deformities of the cleft lip nose. Plast Reconstr Surg. 2002;109:138692, quiz 1393. 